\title{
Cardiovascular Complications in CKD Patients: Role of Oxidative Stress
}

\author{
Elvira O. Gosmanova ${ }^{1}$ and Ngoc-Anh Le $\mathrm{Le}^{2,3}$ \\ ${ }^{1}$ Nephrology Division, Department of Medicine, The University of Tennessee Health Science Center, Memphis, TN 38103, USA \\ ${ }^{2}$ Lipid Research Laboratory, Emory University School of Medicine, Atlanta, GA 30322, USA \\ ${ }^{3}$ Atlanta Veterans Affairs Medical Center, Decatur, GA 30033, USA \\ Correspondence should be addressed to Ngoc-Anh Le, ale@emory.edu
}

Received 14 October 2010; Accepted 8 December 2010

Academic Editor: Ken Ichi Aihara

Copyright ( 2011 E. O. Gosmanova and N.-A. Le. This is an open access article distributed under the Creative Commons Attribution License, which permits unrestricted use, distribution, and reproduction in any medium, provided the original work is properly cited.

\begin{abstract}
Starting with the early stages, patients with chronic kidney disease (CKD) experience higher burden of cardiovascular disease (CVD). Moreover, CVD complications are the major cause of mortality in CKD patients as compared with complications from chronic kidney failure. While traditional CVD risk factors, including diabetes, hypertension, hyperlipidemia, obesity, physical inactivity, may be more prevalent among CKD patients, these factors seem to underestimate the accelerated cardiovascular disease in the CKD population. Search for additional biomarkers that could explain the enhanced CVD risk in CKD patients has gained increasing importance. Although it is unlikely that any single nontraditional risk factor would fully account for the increased CVD risk in individuals with CKD, oxidative stress appears to play a central role in the development and progression of CVD and its complications. We will review the data that support the contribution of oxidative stress in the pathogenesis of CVD in patients with chronic kidney failure.
\end{abstract}

\section{Introduction}

Cardiovascular disease (CVD) is a major cause of morbidity and mortality in patients with chronic kidney disease (CKD) $[1,2]$. Even early stages of CKD that are characterized by relatively preserved or minimally decreased overall renal function are associated with increasing incidence of de novo and recurrent CVD events [3, 4]. As glomerular filtration rate (GFR) diminishes, the prevalence and severity of CVD abnormalities have been reported to be increasing $[5,6]$. Among patients with stages III-IV CKD, the prevalence of CVD is 4- to 5-fold higher than that observed for the general population. CKD patients are known to be affected by diabetes, hypertension, and obesity-which are known traditional CVD risk factors in general population $[7,8]$. However, cross-sectional studies demonstrated that the Framingham Risk Score which is based on the traditional CVD risk factors failed to determine the extent of CVD risk in subjects with CKD and those with end-stage renal disease (ESRD) [9-11], and other factors must be involved in the increased CVD prevalence in this high-risk population $[12,13]$. Before a new biochemical marker could be considered as CVD risk factor, it must meet the following conditions: (a) evidence of the biological plausibility as to why the factor may promote CVD risk; (b) demonstration that the risk factor level increases with severity of kidney disease; (c) evidence of an association between the risk factor and cardiovascular disease in CKD patients; (d) demonstration in double-blind, randomized placebo-controlled clinical trials that treatment of the risk factor decreases CVD outcomes. Available data on the importance of oxidative stress as one of the contributing factors in the prevalence and severity of CVD abnormalities in patients with chronic kidney disease will be summarized in the following sections.

\section{Role of Oxidative Stress in CVD}

Oxidative stress (OxStress) is recognized as a critical factor in the development of atherosclerotic cardiovascular disease 
(ACVD) $[14,15]$. According to the oxidation hypothesis of atherosclerosis, low-density lipoprotein (LDL) in its native state is not atherogenic $[16,17]$. LDL must undergo oxidative modification before it can contribute to the initiation and progression of atherosclerosis [16, 17]. Data from animal models of atherosclerosis, both diet-induced and genetically altered models, have demonstrated the presence of oxidized LDL (oxLDL) in plasma as well as in atherosclerotic lesions $[18,19]$. Presence of oxLDL, autoantibodies against malondialdehyde-modified LDL, and of LDL-IgG immune complexes has also been reported in human plasma and human atherosclerotic lesions $[18,19]$. The pathways involved in the formation of these oxidative markers and the relationship between these markers and disease progression remain to be elucidated.

In case-control studies, some reports have suggested a positive relationship between autoantibodies against MDALDL (one form of oxLDL) and disease severity [20, 21], while others have noted no relationship [22] or inverse relationship [23] between autoantibody levels to oxLDL and the extent of atherosclerosis. In the Watanabe rabbit, animals with high autoantibody levels following immunization with oxLDL were found to have less severe lesions than animals with low antibody levels [24]. We have reported that independent of fasting levels of autoantibodies, patients with diseased endothelium demonstrated a characteristic acute and transient reduction in autoantibody levels following meal consumption [25]. Such a reduction in autoantibody levels was not observed in young healthy controls with one or less than one risk factor. We also reported that this mealinduced reduction in autoantibody levels is unique to meal challenges that are enriched in polyunsaturated fatty acids. Isocaloric meal challenges containing primarily saturated or monounsaturated fatty acids failed to induce the transient reduction in autoantibody levels in these same individuals $[26,27]$.

A large body of evidence suggests that oxidized lowdensity lipoprotein (oxLDL) is the leading candidate in the pathogenesis of atherosclerosis [28] and may serve as a unique mediator of oxidative status in the vascular environment [28]. While the emphasis is on the oxidative modification of LDL, all plasma lipoproteins are subjected to oxidative modification [29]. Case-control studies as well as a limited number of prospective studies have linked the level of oxLDL to disease severity. Due to the heterogeneity of oxLDL and the available epitopes that are recognized by the various antibodies, a number of different immunoassays are available for oxLDL, and the correlation among these measurements is quite poor [30]. Several studies have reported strong and independent relationship between measures of oxLDL and CVD [31-33], including metabolic syndrome [34]. Data from progression/regression studies with nonhuman primates actually complicated the relationship between indices of LDL oxidation and disease status. Using antibodies that recognized the oxidative epitopes of phospholipids moiety of LDL (oxPL), diet-induced atherosclerosis is associated with increased levels of oxLDL and increased levels of total oxPL as well as oxPL/apoB [35]. With regression, as LDL-cholesterol is reduced, there was a modest reduction in total oxPL but a statistically significant increase in oxPL/apoB [35]. In humans, data from clinical trials (REVERSAL, MIRACL) with aggressive LDL-cholesterol reduction also reported and unexpected increase in oxPL/apoB $[36,37]$.

Oxidative modification of LDL resulted in the formation of a lipid-rich particle with specific characteristics that contribute to the development of early atherosclerosis [38]. By inducing adhesion molecules (VCAM-1) and specific receptors, oxLDL stimulates the adhesion of circulating monocytes to endothelial cells [39]. oxLDL can also stimulate the production and release of monocyte chemotactic protein-1 (MCP-1) by endothelial cells and smooth muscle cells resulting in the enhanced migration of monocytes into the arterial intima [40]. oxLDL has also been reported to inhibit in vitro proliferation and survival of vascular cells [41] as well as alterations in the normal function of endothelial cells [42]. Additionally, oxLDL has prothrombotic activity and increases platelet activation and expression of tissue factor and PAI-1 (plasminogen activator inhibitor 1) by endothelial cells [43].

\section{Oxidative Stress in CKD}

Oxidative stress is a state of imbalance between free radicals production and their degradation by antioxidant systems with increased accumulation of the radicals. Reactive oxygen species (ROS) and reactive nitrogen species (RNS) are examples of free radicals. Over $90 \%$ of ROS formation occurs "accidentally" in mitochondria during metabolism of oxygen when some of electrons passing "down" the electron transport chain leak away from the main path and go directly to reduce oxygen molecules to the superoxide anion [44]. In addition, ROS could be "deliberately" synthesized in phagocytic cells, as well as in vascular wall and various other tissues by enzymes such as $\mathrm{NAD}(\mathrm{P}) \mathrm{H}$ oxidase, myeloperoxidase, xanthine oxidase, cyclooxygenase, and lipoxygenase [45]. At low concentrations, ROS (superoxide anion, hydrogen peroxide, hydroxyl radical, hypochlorite ion, and hydroperoxyl radical) involved the vast array of physiologic functions. For example, ROS are known regulators of nitric oxide synthesis, intracellular signaling cascades, including cytokines, growth factors, MAPK, and NF- $\kappa \mathrm{B}$, and also involved in the modulation of immune responses, apoptosis, and mutagenesis [46-48]. Additionally, ROS produced during phagocytic burst is a key defense mechanism against environmental pathogens. However, when ROS are made in excess, they can react with various molecules such as lipids, carbohydrates, proteins, and DNA altering their structure and function, resulting in cellular damage that leads to pathologic processes including, but not limited to, atherosclerosis development. These potentially deleterious reactions are controlled by a system of enzymatic and nonenzymatic antioxidants which eliminate pro-oxidants and scavenge-free radicals. Superoxide dismutase (SOD), glutathione peroxidase, glutathione reductase, and catalase are among the main enzymatic antioxidants. Glutathione, thiols, ascorbic acid, alpha-tocopherol (vitamin E), mixed carotinoids, and bioflavonoids are among the nonenzymatic antioxidant defense processes. 
Direct measurement of free radicals in vivo is difficult due to the highly reactive nature of these compounds and minute concentrations in biological fluids. Instead, we rely on measurement of stable end products of oxidation of different molecules. Numerous biomarkers of oxidative stress have been shown to be elevated in patients with CKD. These include products of lipid oxidation (lipid peroxides, malondialdehyde, and thiobarbituric acid reactive substances) and oxidized LDL $[49,50]$, advanced oxidation protein products (AOPPs) [51], F2 isoprostanes and isolevuglandins (a family of reactive $\gamma$-ketoaldehydes generated by free radical oxidation of arachidonate-containing lipids through the isoprostane pathway) $[52,53]$, and 8-hydroxyl 2 '-deoxyguanosine - marker of oxidative DNA damage [54]. Furthermore, indices of OxStress are increased with severity of kidney disease. For example, glomerular filtration rate has been reported to be inversely associated with levels of AOPP [51], malondialdehyde (MDA) [55], and F2 isoprostanes $[56,57]$, suggesting that decline in renal function may have direct effect on worsening of oxidative stress.

The nature of oxidative stress in chronic kidney disease remains to be elucidated. Impaired oxidative balance in CKD is likely to come from a combination of increased ROS production and reduced clearance as well as an ineffective antioxidant defense mechanism. Several important antioxidant pathways have been reported to be altered in patients with $\mathrm{CKD}$, including reduced erythrocyte SOD activity [55], reduced plasma thiol groups [58], diminished plasma glutathione, and glutathione peroxidase function [59]. However, total antioxidant capacity (TAC) of plasma remains normal or even becomes elevated as CKD progresses [57]. Elevated concentrations of uric acid in CKD patients have been suggested to account for the high capacity for peroxyl radicals that constitute the substrate for the in vitro TAC assay [60].

CKD patients are typically affected by multiple concomitant diseases such as diabetes and hypertension (HTN) which are also associated with oxidative stress $[61,62]$. Increased activity of baseline and stimulated $\mathrm{NAD}(\mathrm{P}) \mathrm{H}$ oxidase are responsible for overproduction of superoxide anion, in circulating peripheral mononuclear (PMN) cells isolated from patients with stage I-II CKD [63]. The presence of CKD appears to further enhance the oxidative stress independently from underlying conditions. Agarwal [64] reported that urinary MDA excretion and protein carbonylation were increased in hypertensive patients with concomitant CKD as compared with patients with HTN and no CKD. The renin-angiotensin-aldosterone system (RAAS) plays an important role in activation of $\mathrm{NAD}(\mathrm{P}) \mathrm{H}$ oxidase in vascular smooth muscle cells and the kidney $[65,66]$. Additionally, NAD $(\mathrm{P}) \mathrm{H}$ oxidase could be activated by cytokines (TNF $\alpha$ ), hyperglycemia, and mechanical stress [65]. Therefore, rigorous studies examining the relation between oxidative stress, CKD, and underline diabetes and HTN are needed in humans to clearly establish if oxidative stress is a marker of severity of underline condition leading to CKD or that CKD independently further promotes OxStress.

Hemodialysis - a procedure utilized as part of usual care in the management of terminal chronic kidney disease, has also been demonstrated to contribute to OxStress. Contact of blood with dialysis membrane during extracorporeal blood purification can lead to PMN cell activation and generation of ROS $[67,68]$. Presence of inadequately removed endotoxin in water used for dialysate preparation also influences PMN activation and ROS production in hemodialysis patients [69]. Myeloperoxidase (MPO) activity has been found to be increased during hemodialysis, especially with the use of bioincompatible dialysis membranes [70]. Moreover, heparin that is routinely used for anticoagulation during hemodialysis is known to activate MPO leading to increased ROS production [71]. Intravenous administered heparin can also displace extracellular-superoxide dismutase (ECSOD) from vascular endothelium [72] by interfering with the binding of the antioxidant enzyme to type $\mathrm{C}$ heparin sulfate proteoglycans. It has been suggested that EC-SOD is the major determinant of nitric oxide bioavailability in blood vessels, and loss of EC-SOD from vascular wall may contribute to endothelial dysfunction [73]. Additionally, plasma ascorbic acid and lipid-soluble alpha-tocopherol, both potent components of an antioxidant defense system, were significantly reduced after single hemodialysis session [74-76]. However, the hemodialysis procedure was also reported to have beneficial effects on total antioxidant capacity by increasing plasma thiol content $[76,77]$.

\section{Association between Oxidative Stress Biomarkers and CVD in CKD Patients}

Strong correlation between oxidative markers and the presence and extent of cardiovascular diseases was found in the general population as outlined in the recent paper [78]. Moreover, oxidative markers were shown to be important predictors of cardiovascular outcomes in prospective analyses [79-83]. Unfortunately, the limited number of studies examined the relationship between oxidative stress markers and cardiovascular disease in patients with kidney failure. Drüeke et al. [84] found that levels of AOPP strongly correlated with carotid artery intima-medial thickness (IMT) in 79 patients with ESRD. Similar finding was reported by Yang et al. in 109 patients with CKD [85]. Additionally, significant positive correlation between carotid artery IMT and TBARS [86] and MPO [87] and negative correlation between carotid artery IMT and reduced SOD and plasma sulfhydryl were reported in patients with ESRD [86]. Shoji et al. [88] observed a statistical trend in correlation between carotid artery IMT and autoantibodies against oxidized LDL. A stronger correlation was observed between femoral artery IMT and autoantibodies against oxidatively modified LDL in ESRD [88].

Prospective studies that examine the association between oxidative stress markers and clinical outcomes in hemodialysis patients are scarce. MPO levels have been shown to predict all-cause mortality in 356 chronic hemodialysis patients [89] with a hazard ratio of 1.14 (95\% confidence interval $1.03-$ 1.26) for each $1,000 \mathrm{pmol} / \mathrm{L}$ increase in MPO level. Interestingly, MPO gene polymorphism was also demonstrated to be associated with presence of CVD and higher CVD-related 
mortality in ESRD patients [90]. Levels of autoantibodies against oxLDL were found to strongly predict cardiovascular mortality during 4 years of follow up in 94 hemodialysis patients [91].

\section{Antioxidant Interventions and Cardiovascular Outcomes in Patients with CKD}

Several small studies have examined the impact of antioxidant interventions on oxidative stress markers in patients with CKD [92-94]. Unfortunately, randomized controlled clinical trials addressing the impact of antioxidant interventions on CVD outcomes in patients with CKD are scarce. The SPACE (Secondary Prevention with Antioxidants of Cardiovascular Disease in End-Stage Renal Disease) was a clinical trial involving 196 patients with ESRD who were randomized to either $800 \mathrm{IU}$ of alpha-tocopherol per day or placebo [95]. During a median followup period of 519 days, statistically significant reduction in the primary composite outcome, consisting of myocardial infarction (fatal and nonfatal), ischemic stroke, peripheral vascular disease, and unstable angina, was observed in patients receiving vitamin E supplementation [95]. The relative risk (RR) was 0.46 (95\% confidence interval (CI) $0.27-0.78$ ) in the vitamin E group as compared to the placebo.

In the second randomized controlled trial, N-acetylcysteine at oral dose $600 \mathrm{mg}$ twice daily over a period of approximately 15 months also significantly reduced primary composite variable consisting of cardiac events including fatal and nonfatal myocardial infarction, cardiovascular disease death, need for coronary angioplasty or coronary bypass surgery, ischemic stroke, peripheral vascular disease with amputation, or need for angioplasty [96]. The relative risk was 0.6 (95\% CI 0.38-0.95). However, no beneficial effects of vitamin $\mathrm{E}$ or $\mathrm{N}$-acetylcysteine administration were observed on all-cause mortality, suggesting that exploration of additional strategies is needed to improve overall survival in dialysis patients. Subgroup analysis of some lipid-lowering studies which have included CKD patients has suggested that statin therapy may also reduce inflammatory and oxidative markers [97]. Additionally, subgroup of patients with CKD taking atorvastatin for a median period of 3.3 years had a statistically significant decrease in cumulative incidence for fatal and nonfatal stroke, total coronary events, total cardiovascular events, and all-cause mortality as compared to placebo in the Anglo-Scandinavian Cardiac Outcomes Trial-Lipid Lowering Arm (ASCOT-LLA) [98]. However, it should also be noted that two large randomized clinical trials using atorvastatin [99] and rosuvastatin [100] failed to demonstrate any reduction of CVD events or all-cause mortality in patients with ESRD. Results from the Study of Heart and Renal Protection (SHARP) might shed more light on cardiovascular benefits of statin use in CKD patients [101]. Additional studies that address the efficacy of novel antioxidants including endogenous antioxidants such as hemeoxygenase-1 and bilirubin $[102,103]$ in reducing oxidative stress CKD patients are needed.
In summary, cardiovascular disease is an important cause of morbidity and mortality in patients with impaired kidney function. Increasing evidence strongly supports oxidative stress as plausible independent cardiovascular risk factor in patients with CKD. Nevertheless, several important questions remain unanswered. The exact processes underlying increased oxidative stress in CKD remain to be elucidated. Furthermore, identification of biochemical and/or functional biomarkers that could be used to monitor oxidative imbalance in CKD may allow the development of optimal intervention strategy to reduce oxidative stress in CKD.

\section{References}

[1] US Renal Data System, USRDS, "Atlas of end-stage renal disease in the United States," Annual Data Report, National Institutes of Health, National Institute of Diabetes and Digestive and Kidney Diseases, Bethesda, Md, USA, 2009.

[2] G. Manjunath, H. Tighiouart, J. Coresh et al., "Level of kidney function as a risk factor for cardiovascular outcomes in the elderly," Kidney International, vol. 63, no. 3, pp. 11211129, 2003.

[3] P. Jungers, Z. A. Massy, T. Nguyen Khoa et al., "Incidence and risk factors of atherosclerotic cardiovascular accidents in predialysis chronic renal failure patients: a prospective study," Nephrology Dialysis Transplantation, vol. 12, no. 12, pp. 2597-2602, 1997.

[4] G. Manjunath, H. Tighiouart, H. Ibrahim et al., "Level of kidney function as a risk factor for atherosclerotic cardiovascular outcomes in the community," Journal of the American College of Cardiology, vol. 41, no. 1, pp. 47-55, 2003.

[5] A. S. Go, G. M. Chertow, D. Fan, C. E. McCulloch, and C. Y. Hsu, "Chronic kidney disease and the risks of death, cardiovascular events, and hospitalization," New England Journal of Medicine, vol. 351, no. 13, pp. 1296-1370, 2004.

[6] B. F. Culleton, M. G. Larson, P. W. F. Wilson, J. C. Evans, P. S. Parfrey, and D. Levy, "Cardiovascular disease and mortality in a community-based cohort with mild renal insufficiency," Kidney International, vol. 56, no. 6, pp. 2214-2219, 1999.

[7] M. J. Sarnak, A. S. Levey, A. C. Schoolwerth et al., "American Heart Association Councils on Kidney in Cardiovascular Disease, High Blood Pressure Research, Clinical Cardiology, and Epidemiology and Prevention.Complications in early CKD. Kidney disease as a risk factor for development of cardiovascular disease: a statement from the American Heart Association Councils on kidney in cardiovascular disease, high blood pressure research, clinical cardiology, and epidemiology and prevention," Circulation, vol. 108, no. 17, pp. 2154-2169, 2003.

[8] M. Goicoechea, S. G. De Vinuesa, F. Gómez-Campderá, and J. Luño, "Predictive cardiovascular risk factors in patients with chronic kidney disease (CKD)," Kidney International, Supplement, vol. 67, no. 93, pp. S35-S38, 2005.

[9] J. C. Longenecker, J. Coresh, N. R. Powe et al., "Traditional cardiovascular disease risk factors in dialysis patients compared with the general population: the CHOICE study," Journal of the American Society of Nephrology, vol. 13, no. 7, pp. 1918-1927, 2002.

[10] A. K. Cheung, M. J. Sarnak, G. Yan et al., "Atherosclerotic cardiovascular disease risks in chronic hemodialysis patients," Kidney International, vol. 58, no. 1, pp. 353-362, 2000. 
[11] P. Muntner, J. He, B. C. Astor, A. R. Folsom, and J. Coresh, "Traditional and nontraditional risk factors predict coronary heart disease in chronic kidney disease: results from the atherosclerosis risk in communities study," Journal of the American Society of Nephrology, vol. 16, no. 2, pp. 529-538, 2005.

[12] D. Laurier, N. P. Chau, B. Gazelles, and P. Segond, "Estimation of CHD risk in a French working population using a modified Framingham model," Journal of Clinical Epidemiology, vol. 47, no. 12, pp. 1353-1364, 1994.

[13] P. M. Ridker, "Novel risk factors and markers for coronary disease," Advances in Internal Medicine, vol. 45, pp. 391-418, 2000.

[14] U. Singh and I. Jialal, "Oxidative stress and atherosclerosis," Pathophysiology, vol. 13, no. 3, pp. 129-142, 2006.

[15] N. R. Madamanchi, A. Vendrov, and M. S. Runge, "Oxidative stress and vascular disease," Arteriosclerosis, Thrombosis, and Vascular Biology, vol. 25, no. 1, pp. 29-38, 2005.

[16] J. L. Witztum, "The oxidation hypothesis of atherosclerosis," Lancet, vol. 344, no. 8925, pp. 793-795, 1994.

[17] M. Torzewski and K. J. Lackner, "Initiation and progression of atherosclerosis-enzymatic or oxidative modification of low-density lipoprotein?" Clinical Chemistry and Laboratory Medicine, vol. 44, no. 12, pp. 1389-1394, 2006.

[18] N.-A. Le, "Reducing oxidized lipids to prevent cardiovascular disease," Current Treatment Options in Cardiovascular Medicine, vol. 10, no. 4, pp. 263-272, 2008.

[19] N. A. Le, "Oxidized lipids and lipoproteins: indices of risk or targets for management," Future Lipidology, vol. 4, no. 1, pp. 41-45, 2009.

[20] J. T. Salonen, S. Ylä-Herttuala, R. Yamamoto et al., "Autoantibody against oxidised LDL and progression of carotid atherosclerosis," Lancet, vol. 339, no. 8798, pp. 883-887, 1992.

[21] A. Boullier, M. Hamon, E. Walters-Laporte et al., "Detection of autoantibodies against oxidized low-density lipoproteins and of IgG-bound low density lipoproteins in patients with coronary artery disease," Clinica Chimica Acta, vol. 238, no. 1, pp. 1-10, 1995.

[22] M. I. J. Uusitupa, L. Niskanen, J. Luoma et al., "Autoantibodies against oxidized LDL do not predict atherosclerotic vascular disease in non-insulin-dependent diabetes mellitus," Arteriosclerosis, Thrombosis, and Vascular Biology, vol. 16, no. 10, pp. 1236-1242, 1996.

[23] L. P. L. Van De Vijver, R. Steyger, G. Van Poppel et al., "Autoantibodies against MDA-LDL in subjects with severe and minor atherosclerosis and healthy population controls," Atherosclerosis, vol. 122, no. 2, pp. 245-253, 1996.

[24] W. Palinski, E. Miller, and J. L. Witztum, "Immunization of low density lipoprotein (LDL) receptor-deficient rabbits with homologous malondialdehyde-modified LDL reduces atherogenesis," Proceedings of the National Academy of Sciences of the United States of America, vol. 92, no. 3, pp. 821$825,1995$.

[25] N.-A. Le, X. Li, S. Kyung, and W. V. Brown, "Evidence for the in vivo generation of oxidatively modified epitopes in patients with atherosclerotic endothelium," Metabolism, vol. 49, no. 10, pp. 1271-1277, 2000.

[26] W. Q. Gradek, M. T. Harris, N. Yahia, W. W. Davis, N. A. Le, and W. V. Brown, "Polyunsaturated fatty acids acutely suppress antibodies to malondialdehyde-modified lipoproteins in patients with vascular disease," American Journal of Cardiology, vol. 93, no. 7, pp. 881-885, 2004.
[27] P. Reaven, S. Parthasarathy, B. J. Grasse, E. Miller, D. Steinberg, and J. L. Witztum, "Effects of oleate-rich and linoleaterich diets on the susceptibility of low density lipoprotein to oxidative modification in mildly hypercholesterolemic subjects," Journal of Clinical Investigation, vol. 91, no. 2, pp. 668-676, 1993.

[28] R. Stocker and J. F. Keaney, "Role of oxidative modifications in atherosclerosis," Physiological Reviews, vol. 84, no. 4, pp. 1381-1478, 2004.

[29] K. Nakajima, T. Nakano, and A. Tanaka, "The oxidative modification hypothesis of atherosclerosis: the comparison of atherogenic effects on oxidized LDL and remnant lipoproteins in plasma," Clinica Chimica Acta, vol. 367, no. 1-2, pp. 36-47, 2006.

[30] S. Tsimikas, "Measures of oxidative otress," Clinics in Laboratory Medicine, vol. 26, no. 3, pp. 571-590, 2006.

[31] P. Holvoet, A. Mertens, P. Verhamme et al., "Circulating oxidized LDL is a useful marker for identifying patients with coronary artery disease," Arteriosclerosis, Thrombosis, and Vascular Biology, vol. 21, no. 5, pp. 844-848, 2001.

[32] K. Wallenfeldt, B. Fagerberg, J. Wikstrand, and J. Hulthe, "Oxidized low-density lipoprotein in plasma is a prognostic marker of subclinical atherosclerosis development in clinically healthy men," Journal of Internal Medicine, vol. 256, no. 5, pp. 413-420, 2004.

[33] P. Holvoet, N. S. Jenny, P. J. Schreiner, R. P. Tracy, and D. R. Jacobs, "The relationship between oxidized LDL and other cardiovascular risk factors and subclinical CVD in different ethnic groups: the Multi-Ethnic Study of Atherosclerosis (MESA)," Atherosclerosis, vol. 194, no. 1, pp. 245-252, 2007.

[34] P. Holvoet, D. H. Lee, M. Steffes, M. Gross, and D. R. Jacobs, "Association between circulating oxidized low-density lipoprotein and incidence of the metabolic syndrome," Journal of the American Medical Association, vol. 299, no. 19, pp. 2287-2293, 2008.

[35] S. Tsimikas, M. Aikawa, F. J. Miller Jr. et al., "Increased plasma oxidized phospholipid:apolipoprotein B-100 ratio with concomitant depletion of oxidized phospholipids from atherosclerotic lesions after dietary lipid-lowering: a potential biomarker of early atherosclerosis regression," Arteriosclerosis, Thrombosis, and Vascular Biology, vol. 27, no. 1, pp. 175-181, 2007.

[36] S. H. Choi, A. Chae, E. Miller et al., "Relationship between Biomarkers of oxidized LDL, tstain therapy, quantitative coronary angiography, and atheroma volume," Journal of the American College of Cardiology, vol. 52, no. 1, pp. 24-32, 2008.

[37] A. E. Fraley, G. G. Schwartz, A. G. Olsson et al., "Relationship of oxidized phospholipids and biomarkers of oxLDL with cardiovascular risk factors, inflammatory biomarkers, and effect of statin therapy in patients with acute coronary syndromes," Journal of the American College of Cardiology, vol. 53, no. 23, pp. 2186-2196, 2009.

[38] D. Steinberg, S. Parthasarathy, T. E. Carew, J. C. Khoo, and J. L. Witztum, "Beyond cholesterol: modifications of lowdensity lipoprotein that increase its atherogenicity," New England Journal of Medicine, vol. 320, no. 14, pp. 915-924, 1989.

[39] J. A. Berliner, M. C. Territo, A. Sevanian et al., "Minimally modified low density lipoprotein stimulates monocyte endothelial interactions," Journal of Clinical Investigation, vol. 85, no. 4, pp. 1260-1266, 1990. 
[40] S. D. Cushing, J. A. Berliner, A. J. Valente et al., "Minimally modified low density lipoprotein induces monocyte chemotactic protein 1 in human endothelial cells and smooth muscle cells," Proceedings of the National Academy of Sciences of the United States of America, vol. 87, no. 13, pp. 5134-5138, 1990.

[41] J. Schuh, A. Novogrodsky, and R. H. Haschemeyer, "Inhibition of lymphocyte mitogenesis by autoxidized low-density lipoprotein," Biochemical and Biophysical Research Communications, vol. 84, no. 3, pp. 763-768, 1978.

[42] J. E. Triau, S. N. Meydani, and E. J. Schaefer, "Oxidized low density lipoprotein stimulates prostacyclin production by adult human vascular endothelial cells," Arteriosclerosis, vol. 8, no. 6, pp. 810-818, 1988.

[43] K. R. Bruckdorfer, "The influence of oxidized lipoproteins, oxidation products and antioxidants on the release of nitric oxide from the endothelium and the response of platelets to nitric oxide," BioFactors, vol. 6, no. 2, pp. 191-199, 1997.

[44] S. R. Pieczenik and J. Neustadt, "Mitochondrial dysfunction and molecular pathways of disease," Experimental and Molecular Pathology, vol. 83, no. 1, pp. 84-92, 2007.

[45] M. Valko, D. Leibfritz, J. Moncol, M. T. D. Cronin, M. Mazur, and J. Telser, "Free radicals and antioxidants in normal physiological functions and human disease," International Journal of Biochemistry and Cell Biology, vol. 39, no. 1, pp. 44-84, 2007.

[46] W. Dröge, "Free radicals in the physiological control of cell function," Physiological Reviews, vol. 82, no. 1, pp. 47-95, 2002.

[47] T. Finkel, "Oxidant signals and oxidative stress," Current Opinion in Cell Biology, vol. 15, no. 2, pp. 247-254, 2003.

[48] C. Nathan, "Specificity of a third kind: reactive oxygen and nitrogen intermediates in cell signaling," Journal of Clinical Investigation, vol. 111, no. 6, pp. 769-778, 2003.

[49] R. Agarwal, "Chronic kidney disease is associated with oxidative stress independent of hypertension," Clinical Nephrology, vol. 61, no. 6, pp. 377-383, 2004.

[50] S. H. A. Diepeveen, G. H. W. E. Verhoeven, J. Van Der Palen et al., "Oxidative stress in patients with end-stage renal disease prior to the start of renal replacement therapy," NephronClinical Practice, vol. 98, no. 1, pp. c3-c7, 2004.

[51] V. Witko-Sarsat, M. Friedlander, T. N. Khoa et al., "Advanced oxidation protein products as novel mediators of inflammation and monocyte activation in chronic renal failure," Journal of Immunology, vol. 161, no. 5, pp. 2524-2532, 1998.

[52] T. A. Ikizler, J. D. Morrow, L. J. Roberts et al., "Plasma F-isoprostane levels are elevated in chronic hemodialysis patients," Clinical Nephrology, vol. 58, no. 3, pp. 190-197, 2002.

[53] R. G. Salomon, E. Batyreva, K. Kaur et al., "Isolevuglandinprotein adducts in humans: products of free radical-induced lipid oxidation through the isoprostane pathway," Biochimica et Biophysica Acta-Molecular and Cell Biology of Lipids, vol. 1485, no. 2-3, pp. 225-235, 2000.

[54] M. P. Montesa, M. G. Rico, M. S. Salguero et al., "Study of oxidative stress in advanced kidney diseaseEstudio del estrés oxidativo en enfermedad renal avanzada," Nefrologia, vol. 29, no. 5, pp. 464-473, 2009.

[55] M. I. Yilmaz, M. Saglam, K. Caglar et al., "The determinants of endothelial dysfunction in CKD: oxidative stress and asymmetric dimethylarginine," American Journal of Kidney Diseases, vol. 47, no. 1, pp. 42-50, 2006.

[56] S. Cottone, G. Mulè, M. Guarneri et al., "Endothelin-1 and F2-isoprostane relate to and predict renal dysfunction in hypertensive patients," Nephrology Dialysis Transplantation, vol. 24, no. 2, pp. 497-503, 2009.

[57] E. Dounousi, E. Papavasiliou, A. Makedou et al., "Oxidative stress is progressively enhanced with advancing stages of CKD," American Journal of Kidney Diseases, vol. 48, no. 5, pp. 752-760, 2006.

[58] J. Himmelfarb, E. McMenamin, and E. McMonagle, "Plasma aminothiol oxidation in chronic hemodialysis patients," Kidney International, vol. 61, no. 2, pp. 705-716, 2002.

[59] I. Ceballos-Picot, V. Witko-Sarsat, M. Merad-Boudia et al., "Glutathione antioxidant system as a marker of oxidative stress in chronic renal failure," Free Radical Biology and Medicine, vol. 21, no. 6, pp. 845-853, 1996.

[60] T. Nguyen-Khoa, Z. A. Massy, V. Witko-Sarsat et al., "Critical evaluation of plasma and LDL oxidant-trapping potential in hemodialysis patients," Kidney International, vol. 56, no. 2, pp. 747-753, 1999.

[61] A. C. Maritim, R. A. Sanders, and J. B. Watkins, "Diabetes, oxidative stress, and antioxidants: a review," Journal of Biochemical and Molecular Toxicology, vol. 17, no. 1, pp. 2438, 2003.

[62] J. De Champlain, R. Wu, H. Girouard et al., "Oxidative stress in hypertension," Clinical and Experimental Hypertension, vol. 26, no. 7-8, pp. 593-601, 2004.

[63] A. Fortuño, O. Beloqui, G. San José, M. U. Moreno, G. Zalba, and J. Díez, "Increased phagocytic nicotinamide adenine dinucleotide phosphate oxidase-dependent superoxide production in patients with early chronic kidney disease," Kidney International. Supplement, no. 99, pp. S71-S75, 2005.

[64] R. Agarwal, "Chronic kidney disease is associated with oxidative stress independent of hypertension," Clinical Nephrology, vol. 61, no. 6, pp. 377-383, 2004.

[65] R. P. Brandes and J. Kreuzer, "Vascular NADPH oxidases: molecular mechanisms of activation," Cardiovascular Research, vol. 65, no. 1, pp. 16-27, 2005.

[66] K. Miyata, M. Rahman, T. Shokoji et al., "Aldosterone stimulates reactive oxygen species production through activation of NADPH oxidase in rat mesangial cells," Journal of the American Society of Nephrology, vol. 16, no. 10, pp. 29062912, 2005.

[67] M. Kosch, A. Lever, M. Fobker et al., "Dialysis filter type determines the acute of haemodialysis on endothelial function and oxidative stress," Nephrology Dialysis Transplantation, vol. 18, no. 7, pp. 1370-1375, 2003.

[68] J. Himmelfarb, K. A. Ault, D. Holbrook, D. A. Leeber, and R. M. Hakim, "Intradialytic granulocyte reactive oxygen species production: a prospective, crossover trial," Journal of the American Society of Nephrology, vol. 4, no. 2, pp. 178-186, 1993.

[69] Y. Oka, M. Miyazaki, S. Takatsu et al., "Lowering of oxidative stress in hemodialysis patients by dialysate cleaning: in relation to arteriosclerosis," Therapeutic Apheresis and Dialysis, vol. 8, no. 4, pp. 313-319, 2004.

[70] C. C. Wu, J. S. Chen, W. M. Wu et al., "Myeloperoxidase serves as a marker of oxidative stress during single haemodialysis session using two different biocompatible dialysis membranes," Nephrology Dialysis Transplantation, vol. 20, pp. 1134-1139, 2005.

[71] M. Gritters, M. P. C. Grooteman, M. Schoorl et al., "Citrate anticoagulation abolishes degranulation of polymorphonuclear cells and platelets and reduces oxidative stress during haemodialysis," Nephrology Dialysis Transplantation, vol. 21, no. 1, pp. 153-159, 2006. 
[72] F. M. Faraci and S. P. Didion, "Vascular protection: superoxide dismutase isoforms in the vessel wall," Arteriosclerosis, Thrombosis, and Vascular Biology, vol. 24, no. 8, pp. 13671373, 2004.

[73] T. Fukai, R. J. Folz, U. Landmesser, and D. G. Harrison, "Extracellular superoxide dismutase and cardiovascular disease," Cardiovascular Research, vol. 55, no. 2, pp. 239-249, 2002.

[74] J. F. Sullivan and A. B. Eisenstein, "Ascorbic acid depletion in patients undergoing chronic hemodialysis," American Journal of Clinical Nutrition, vol. 23, no. 10, pp. 1339-1346, 1970.

[75] M. Morena, J. P. Cristol, J. Y. Bosc et al., "Convective and diffusive losses of vitamin $\mathrm{C}$ during haemodiafiltration session: a contributive factor to oxidative stress in haemodialysis patients," Nephrology Dialysis Transplantation, vol. 17, no. 3, pp. 422-427, 2002.

[76] P. Jackson, C. M. Loughrey, J. H. Lightbody, P. T. McNamee, and I. S. Young, "Effect of hemodialysis on total antioxidant capacity and serum antioxidants in patients with chronic renal failure," Clinical Chemistry, vol. 41, no. 8, part 1, pp. 1135-1138, 1995.

[77] J. Himmelfarb, E. McMonagle, and E. McMenamin, "Plasma protein thiol oxidation and carbonyl formation in chronic renal failure," Kidney International, vol. 58, no. 6, pp. 2571$2578,2000$.

[78] S. Tsimikas, "Oxidative stress in the diagnosis and prognosis of cardiovascular disease," American Journal of Cardiology, vol. 98, no. 11A, pp. 9P-17P, 2006.

[79] S. Tsimikas, S. Kiechl, J. Willeit et al., "Oxidized Phospholipids Predict the Presence and Progression of Carotid and Femoral Atherosclerosis and Symptomatic Cardiovascular Disease. Five-Year Prospective Results From the Bruneck Study," Journal of the American College of Cardiology, vol. 47, no. 11, pp. 2219-2228, 2006.

[80] M. Exner, E. Minar, W. Mlekusch et al., "Myeloperoxidase predicts progression of carotid stenosis in states of low high-density lipoprotein cholesterol," Journal of the American College of Cardiology, vol. 47, no. 11, pp. 2212-2218, 2006.

[81] S. Tsimikas, Z. Mallat, P. J. Talmud et al., "Oxidationspecific biomarkers, lipoprotein(a), and risk of fatal and nonfatal coronary events," Journal of the American College of Cardiology, vol. 56, no. 12, pp. 946-955, 2010.

[82] N. Ahmadi, S. Tsimikas, F. Hajsadeghi et al., "Relation of oxidative biomarkers, vascular dysfunction, and progression of coronary artery calcium," American Journal of Cardiology, vol. 105, no. 4, pp. 459-466, 2010.

[83] S. Kiechl, J. Willeit, M. Mayr et al., "Oxidized phospholipids, lipoprotein(a), lipoprotein-associated phospholipase A2 Activity, and 10-year cardiovascular outcomes: prospective results from the bruneck study," Arteriosclerosis, Thrombosis, and Vascular Biology, vol. 27, no. 8, pp. 17881795, 2007.

[84] T. Drüeke, V. Witko-Sarsat, Z. Massy et al., "Iron therapy, advanced oxidation protein products, and carotid artery intima-media thickness in end-stage renal disease," Circulation, vol. 106, no. 17, pp. 2212-2217, 2002.

[85] X. B. Yang, F. F. Hou, Q. Wu et al., "AOPP level correlate with CA intima thickness: [Increased levels of advanced oxidation protein products are associated with atherosclerosis in chronic kidney disease]," Zhonghua Nei Ke Za Zhi, vol. 44, no. 5, pp. 342-346, 2005.

[86] B. Dursun, E. Dursun, G. Suleymanlar et al., "Carotid artery intima-media thickness correlates with oxidative stress in chronic haemodialysis patients with accelerated atherosclerosis," Nephrology Dialysis Transplantation, vol. 23, no. 5, pp. 1697-1703, 2008.

[87] H. Honda, M. Ueda, S. Kojima et al., "Assessment of myeloperoxidase and oxidative $\alpha$-antitrypsin in patients on hemodialysis," Clinical Journal of the American Society of Nephrology, vol. 4, no. 1, pp. 142-151, 2009.

[88] T. Shoji, E. Kimoto, K. Shinohara et al., "The association of antibodies against oxidized low-density lipoprotein with atherosclerosis in hemodialysis patients," Kidney International, Supplement, vol. 63, no. 84, pp. S128-S130, 2003.

[89] K. Kalantar-Zadeh, M. L. Brennan, and S. L. Hazen, "Serum Myeloperoxidase and Mortality in Maintenance Hemodialysis Patients," American Journal of Kidney Diseases, vol. 48, no. 1, pp. 59-68, 2006.

[90] D. A. Grahl, J. Axelsson, L. Nordfors et al., "Associations between the CYBA 242C/T and the MPO -463G/A polymorphisms, oxidative stress and cardiovascular disease in chronic kidney disease patients," Blood Purification, vol. 25, no. 2, pp. 210-218, 2007.

[91] B. Bayés, M. C. Pastor, J. Bonal, A. Foraster, and R. Romero, "Oxidative stress, inflammation and cardiovascular mortality in haemodialysis-role of seniority and intravenous ferrotherapy: analysis at 4 years of follow-up," Nephrology Dialysis Transplantation, vol. 21, no. 4, pp. 984-990, 2006.

[92] M. Mune, S. Yukawa, M. Kishino et al., "Effect of vitamin E on lipid metabolism and atherosclerosis in ESRD patients," Kidney International, Supplement, vol. 56, no. 71, pp. S126S129, 1999.

[93] K. N. Islam, D. O’Byrne, S. Devaraj, B. Palmer, S. M. Grundy, and I. Jialal, "Alpha-tocopherol supplementation decreases the oxidative susceptibility of LDL in renal failure patients on dialysis therapy," Atherosclerosis, vol. 150, no. 1, pp. 217-224, 2000.

[94] V. Witko-Sarsat, V. Gausson, A. T. Nguyen et al., "AOPPinduced activation of human neutrophil and monocyte oxidative metabolism: a potential target for $\mathrm{N}$-acetylcysteine treatment in dialysis patients," Kidney International, vol. 64, no. 1, pp. 82-91, 2003.

[95] M. Boaz, S. Smetana, T. Weinstein et al., "Secondary prevention with antioxidants of cardiovascular disease in endstage renal disease (SPACE): randomised placebo-controlled trial," Lancet, vol. 356, no. 9237, pp. 1213-1218, 2000.

[96] M. Tepel, M. Van der Giet, M. Statz, J. Jankowski, and W. Zidek, "The antioxidant acetylcysteine reduces cardiovascular events in patients with end-stage renal failure: a randomized, controlled trial," Circulation, vol. 107, no. 7, pp. 992-995, 2003.

[97] V. Cachofeiro, M. Goicochea, S. G. de Vinuesa, P. Oubiña, V. Lahera, and J. Luño, "Oxidative stress and inflammation, a link between chronic kidney disease and cardiovascular disease," Kidney International. Supplement, no. 111, pp. S4S9, 2008

[98] P. S. Sever, B. Dahlöf, N. R. Poulter et al., "Prevention of coronary and stroke events with atorvastatin in hypertensive patients who have average or lower-than-average cholesterol concentrations, in the Anglo-Scandinavian Cardiac Outcomes Trial-Lipid Lowering Arm (ASCOT-LLA): a multicentre randomised controlled trial," Lancet, vol. 361, no. 9364, pp. 1149-1158, 2003.

[99] C. Wanner and V. Krane, "Lessons learnt from the 4D trial," Nephrologie et Therapeutique, vol. 2, no. 1, pp. 3-7, 2006.

[100] B. C. Fellström, A. G. Jardine, R. E. Schmieder et al., "Rosuvastatin and cardiovascular events in patients undergoing 
hemodialysis," New England Journal of Medicine, vol. 360, no. 14, pp. 1395-1407, 2009.

[101] SHARP Collaborative Group, "Study of Heart and Renal Protection (SHARP): randomized trial to assess the effects of lowering low-density lipoprotein cholesterol among 9,438 patients with chronic kidney disease," American Heart Journal, vol. 160, no. 5, pp. 785-794, 2010.

[102] M. F. McCarty, "”Iatrogenic Gilbert syndrome”-a strategy for reducing vascular and cancer risk by increasing plasma unconjugated bilirubin," Medical Hypotheses, vol. 69, no. 5, pp. 974-994, 2007.

[103] M. Fujii, T. Inoguchi, S. Sasaki et al., "Bilirubin and biliverdin protect rodents against diabetic nephropathy by downregulating $\mathrm{NAD}(\mathrm{P}) \mathrm{H}$ oxidase," Kidney International, vol. 78, no. 9, pp. 905-919, 2010. 


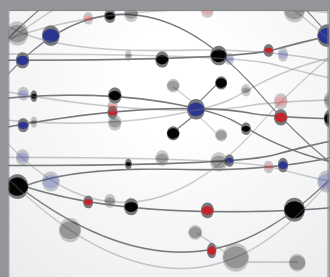

The Scientific World Journal
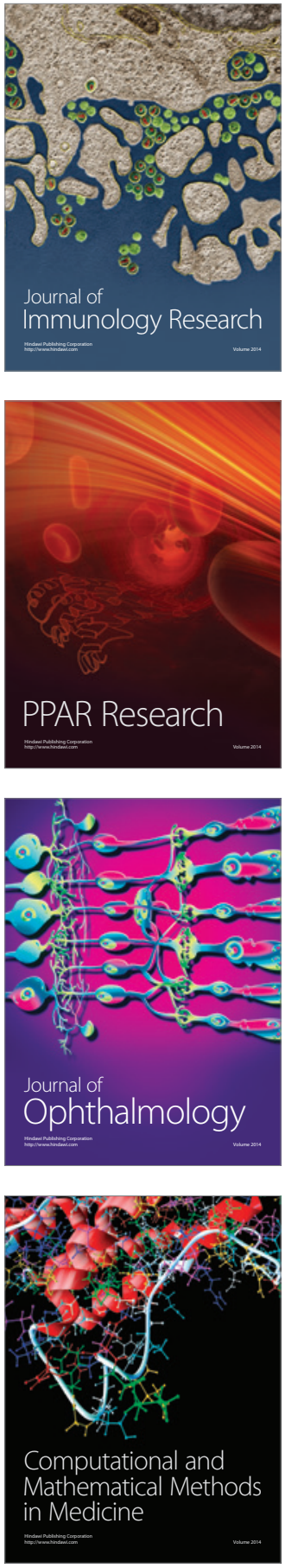

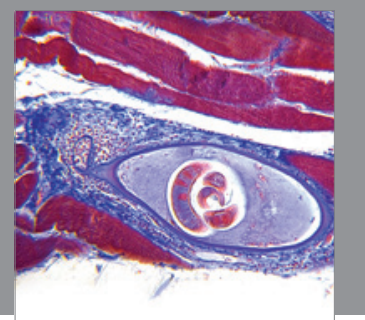

Gastroenterology

Research and Practice
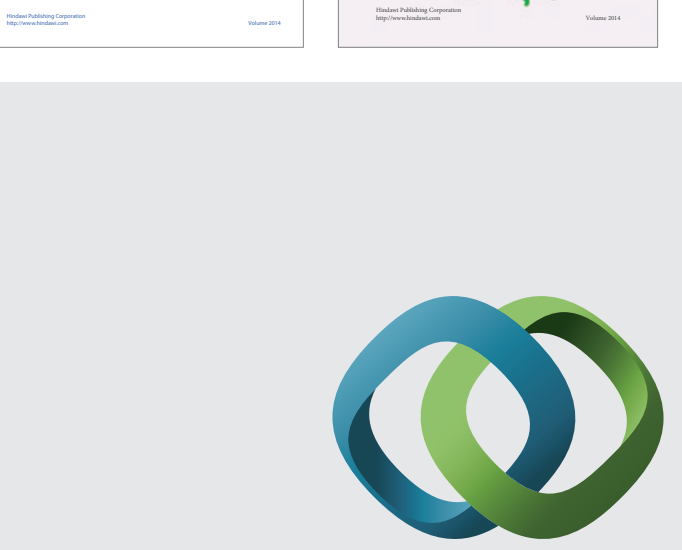

\section{Hindawi}

Submit your manuscripts at

http://www.hindawi.com
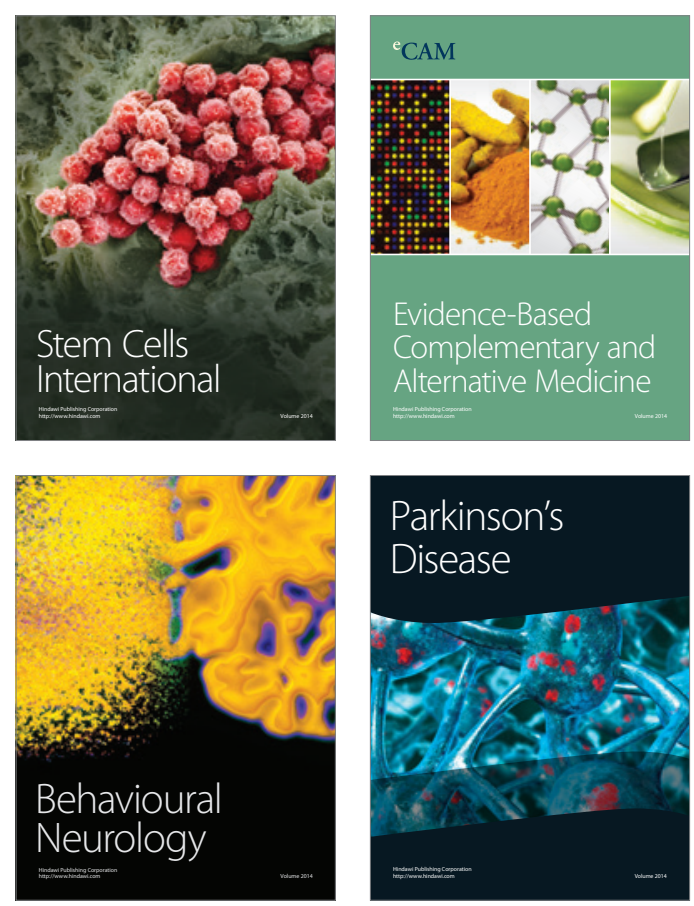

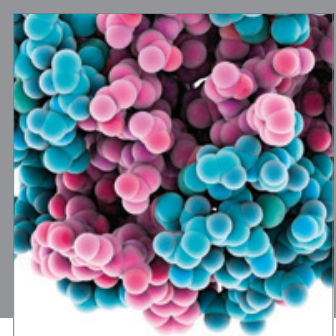

Journal of
Diabetes Research

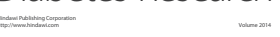

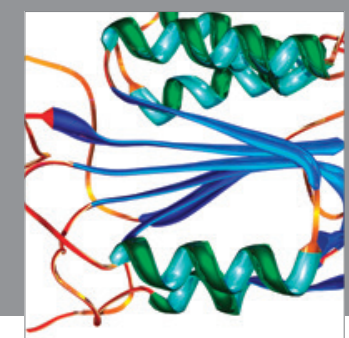

Disease Markers
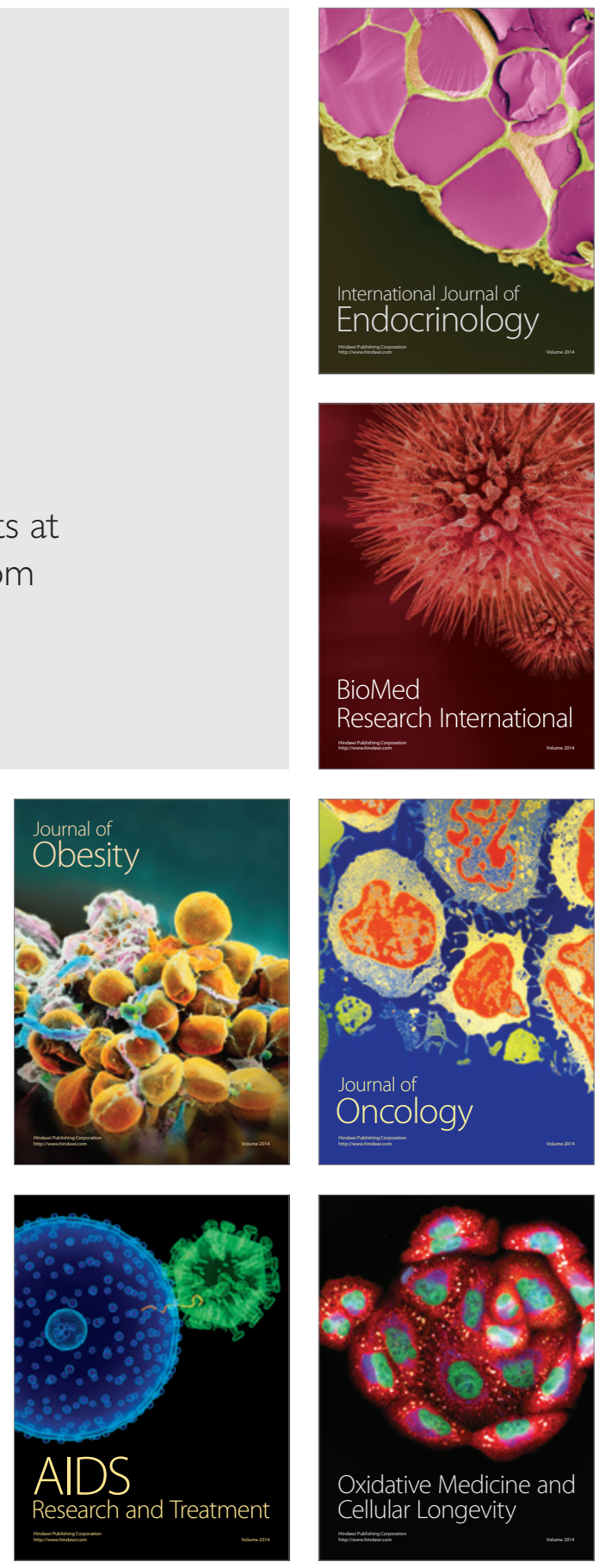